\title{
Nontransitive dice sets realizing Paley tournaments for solving Schütte's tournament problem
}

\author{
S. Bozóki
}




\title{
NONTRANSITIVE DICE SETS REALIZING THE PALEY TOURNAMENTS FOR SOLVING SCHÜTTE'S TOURNAMENT PROBLEM
}

\author{
S. BOZÓKI \\ Received 05 December, 2012
}

\begin{abstract}
The problem of a multiple player dice tournament is discussed and solved in the paper. A die has a finite number of faces with real numbers written on each. Finite dice sets are proposed which have the following property, defined by Schütte for tournaments: for an arbitrary subset of $k$ dice there is at least one die that beats each of the $k$ with a probability greater than $1 / 2$. It is shown that the proposed dice set realizes the Paley tournament, that is known to have the Schütte property (for a given $k$ ) if the number of vertices is large enough. The proof is based on Dirichlet's theorem, stating that the sum of quadratic nonresidues is strictly larger than the sum of quadratic residues.
\end{abstract}

2010 Mathematics Subject Classification: 05C20; 05C69; 11A15

Keywords: nontransitive binary relation, the Paley tournament, the Schütte property, quadratic residues

\section{INTRODUCTION}

The game of rock, paper, scissors is one of the simplest nontransitive tournaments. The relation between any two elements from the set \{rock, paper, scissors\} is well defined. However, the directed graph of three vertices - representing the three objects, with edges leading from rock to scissors (since rock beats scissors), from scissors to paper, and from paper to rock -is a directed three-cycle. In other words, there is no optimal choice among the three alternatives, since any of them is beaten by another one. This game is directly defined to be a paradox.

Similar types of nontransitivity can be observed with certain dice sets. Let us have a finite set of dice, each die having a finite number of faces (not necessarily with the same probabilities) and each face having a real number written upon it. Die $i$ beats die $j$ if, when rolled together repeatedly, the number of cases when the rolled face of die $i$ is greater than the rolled face of die $j$ is, in the long run, greater than the half of the number of total cases. In terms of probability theory, dice are considered as independent discrete random variables, and die $X$ beats die $Y$ if $P(X>Y)>1 / 2$.

The author was supported in part by OTKA grant K 77420 . 
Definition 1. (see, e.g., [28, Def. 5.3]) Let $G$ be a tournament on $n$ vertices. Dice set $\left\{X_{1}, X_{2}, \ldots, X_{n}\right\}$ realizes $G$ if $P\left(X_{i}>X_{j}\right)>1 / 2$ if and only if $i \rightarrow j$ is an edge of $G$.

Section 2 introduces quadratic residues, the Paley tournament and the Schütte property. The Paley tournament [21], denoted by $P_{p}$ hereafter, is a complete directed graph of $p$ vertices without loops, where $p=4 m+3 \geq 7$ is a prime number. If $j-i$ is a quadratic residue modulo $p$, then a directed edge is drawn from vertex $i$ to vertex $j$, otherwise it is drawn in the opposite direction. Note that the Paley tournament can be defined not only for prime numbers, and not only for prime numbers in the form of $p=4 m+3$, however, those variants are not used in the paper.

For a given positive integer $k$, the Schütte property, denoted by $S_{k}$, is defined for directed graphs, and, as a special case, it is applicable to tournaments: for every $k$ vertices there exists at least one vertex, from which edges go to each of the $k$ vertices [10]. It follows from the definition that a tournament with property $S_{k}$ fulfills property $S_{k^{\prime}}$ as well, when $k>k^{\prime}$.

Several lower and upper bounds are known for the minimal number $n$ such that there exists a tournament on $n$ vertices that fulfills property $S_{k}$. One of them is directly applied in the paper, provided by Graham and Spencer [14]: if $p>k^{2} 2^{2 k-2}$, then $P_{p}$ fulfills property $S_{k}$.

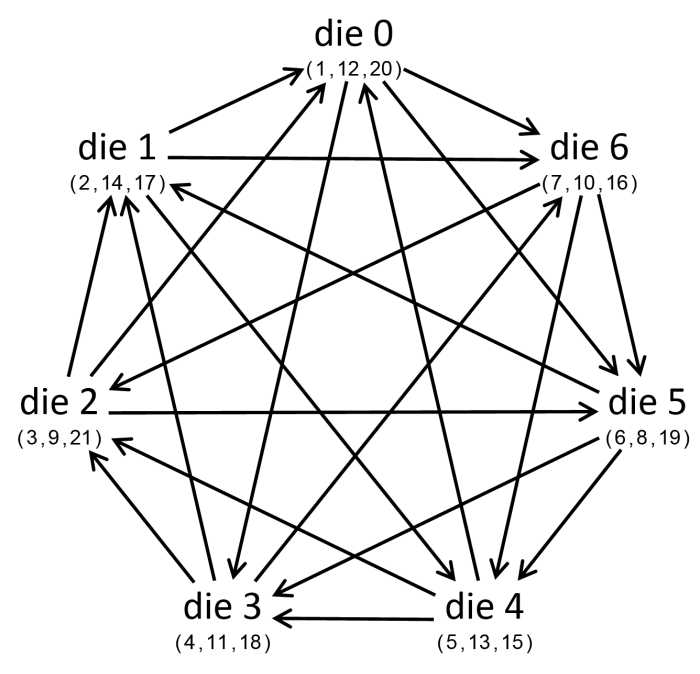

FIgURE 1. van Deventer's dice set realizes $P_{7}$.

Section 3 summarizes a number of nontransitive dice sets, starting with the one by Steinhaus and Trybula [30]. The multiple player dice tournament problem requires property $S_{k}$ : for every $k$ dice there exists at least one die in the set that beats each of the $k$. A nontransitive dice set for three players (having property $S_{2}$ ) is proposed by 
van Deventer [5]. Each die in Figure 1 has three faces with equal probabilities, and the numbers written on them are given in parentheses. A directed edge is going from die $i$ to die $j$ if and only if die $i$ wins over die $j$ with probability greater than $1 / 2$, the tournament resulted in is $P_{7}$, that is, $P_{7}$ is realized by the dice set in Figure 1 .

Departing from van Deventer's dice set, the results of the paper are presented in Section 4. Dice set $D_{p}$ with $p$ dice is proposed for all $p=4 m+3(m \geq 1)$ prime numbers and it shown that $D_{p}$ realizes $P_{p}$.

In terms of probability theory again, for any $k$, a constructive definition of the set of independent discrete random variables $X_{1}, X_{2}, \ldots, X_{p}$ is given such that each variable of an arbitrarily chosen subset $X_{i_{1}}, X_{i_{2}}, \ldots, X_{i_{k}}$ can be simultaneously beaten by at least one of the other variables. That is, there exists a random variable $X_{i_{k+1}}$ in the set such that $P\left(X_{i_{k+1}}>X_{i_{1}}\right)>1 / 2, P\left(X_{i_{k+1}}>X_{i_{2}}\right)>1 / 2, \ldots, P\left(X_{i_{k+1}}>\right.$ $\left.X_{i_{k}}\right)>1 / 2$. Moreover, as an unintended secondary result, all probabilities above are equal.

Section 5 concludes and discusses further research of realizations having fewer faces and possible relations to voting theory.

\section{QUADRATIC RESIDUES, SCHÜTTE'S TOURNAMENT PROBLEM AND THE PALEY TOURNAMENTS}

Let $p=4 m+3(m \geq 1)$ be a prime number. $q$ is called a quadratic residue modulo $p$, if $q$ is congruent to a perfect square modulo $p$-otherwise it is called a quadratic nonresidue. Three properties of quadratic residues are applied in the paper. The first two properties refer to the sets of quadratic residues and of quadratic nonresidues (see, e.g., Theorem 3.1. in the book of Niven and Zuckerman [20]). If $q$ is a quadratic residue modulo $p$, then $p-q$ is a quadratic nonresidue, and vice versa. The set of nonzero quadratic residues is closed for multiplication (modulo $p$ ) by any nonzero quadratic residue (and the set of quadratic nonresidues is closed for multiplication by any nonzero quadratic residue). The third property is related to the distribution of quadratic residues proven by Dirichlet [6-8], starting from Jacobi's observation [17]:

Theorem 1. For $p \geq 7$, the sum of the residues minus the sum of the nonresidues is a negative number, and it is an odd multiple of $p$.

Probably the shortest proof of Theorem 1 is due to Moser [19]. For the illustrative example $p=7$, the sum of quadratic residues is $(0+) 1+2+4=7$, while the sum of quadratic nonresidues is $3+5+6=14$, and their difference is $7-14=-1 \cdot 7$.

Erdôs writes in one of his more than twenty papers from 1963 [10, p. 221]: 
"The problem was recently put to me by Professor Schütte in its graph-theoretic form: If $\mathscr{E}^{(n)}$ is a complete directed graph, with $n$ vertices, which has the property that for every $k$ vertices of $\mathscr{G}^{(n)}$ there is at least one vertex from which edges go out to each of the $k$, we shall say that $E^{(n)}$ has the property $S_{k}$. Schütte's problem is to show that for every $k$ there is a $g^{(n)}$ with the property $S_{k}$ and to find the least possible n for a given $k$."

Let us keep the notations above and let $f(k)$ denote the minimal number of $n$ such that there exists a tournament on $n$ vertices having property $S_{k}$.

That $f(k)<\infty$ (for all $k=1,2, \ldots$ ) is proved first by Erdôs [10], using the probabilistic method, while a geometric proof is given by Alon, Brightwell, Kierstead, Kostochka and Winkler [1]. It can be noted that Schütte's problem has several variants, e.g., a bipartite version discussed by Borowiecki, Grytczuk, Haluszczak and Tuza [4].

Lower and upper bounds for $f(k)$ are also known:

$$
\begin{aligned}
f(k) & \geq 2^{k+1}-1 \quad \text { (Erdős [10, p. 221]) } \\
f(k) & \geq 2^{k-1}(k+2)-1 \quad \text { (Szekeres and Szekeres [32, p. 292]) } \\
\underset{k}{\limsup \frac{f(k)}{2^{k} k^{2}}} & \geq \log 2 \quad(\text { Erdôs [10, p. 221]) }
\end{aligned}
$$

However, exact values of $f(k)$ are known only for small $k$. Szekeres and Szekeres [32, p. 293] showed that $f(1)=3, f(2)=7, f(3)=19$.

Definition 2. Let $p=4 m+3 \geq 7$ be a prime number. The Paley tournament on $p$ vertices, denoted by $P_{p}$, is defined by its incidence matrix $\mathbf{M}$ of size $p \times p$ as follows:

$$
\mathbf{M}_{i, j}:=\left\{\begin{aligned}
0 & \text { if } i=j, \\
1 & \text { if } i-j \text { is a quadratic residue modulo } p, \\
-1 & \text { if } j-i \text { is a quadratic residue modulo } p .
\end{aligned}\right.
$$

Following from the properties of quadratic residues, $P_{p}$ is well defined. It is a simple complete directed graph of $p$ vertices (without loops), in which there is a directed edge from vertex $i$ to vertex $j$ if and only if $j-i$ is a quadratic residue modulo $p$. Otherwise (that is, if $i-j$ is a quadratic residue) the directed edge goes from vertex $j$ to vertex $i$. A rotational symmetry of $P_{p}$ can be also observed: the $(i+1)$-th row is a shifted copy of the $i$-th row, and the last element is relocated to the first position.

The construction is originally given in a linear algebraic way by Paley [21], and, according to Goethals and Seidel [13, p. 1002], it is represented as graphs by Sachs [26] and Erdôs and Rényi [11]. Graham and Spencer [14] draw $P_{p}$ as directed graph and give a sufficient condition for fulfilling $S_{k}$ :

Theorem 2. If $p>k^{2} 2^{2 k-2}$, then $P_{p}$ has property $S_{k}$. 
Graham and Spencer [14, p. 47] also propose that $P_{67}$ has property $S_{4}$ and so, consequently, $f(4) \leq 67$. Their conjecture is that $f(4)=67$. According to Reid, McRae, Hedetniemi and Hedetniemi [24] it was computationally verified by Fisher in 1996 that

Proposition 1. Among the Paley tournaments:

(1) $P_{67}$ is the smallest one having property $S_{4}$;

(2) $P_{331}$ is the smallest one having property $S_{5}$;

(3) $P_{1163}$ is the smallest one having property $S_{6}$.

Fisher's results are published in [24] as private communication. We tried to confirm Fisher's results and succeeded for the first two regarding $S_{4}$ and $S_{5}$. However, the third result was only confirmed in part: it was shown that $P_{p}$ does not have property $S_{6}$ if $4 m+3=p<1163$ is a prime number.

Tyszkiewicz [36] proposed tournaments fulfilling property $S_{k}$ and they are different from the Paley tournaments.

\section{NONTRANSITIVE DICE SETS}

Steinhaus and Trybula [30, p. 69] constructed three independent random variables $X, Y, Z$ as follows: let $c>4$ be an arbitrary constant and $P(X=c-5 / 3)=$ $3 / 8, P(X=c+1)=5 / 8, P(Y=c+5 / 3)=3 / 8, P(Y=c-1)=5 / 8, P(Z=$ $c-2 / 3 \sqrt{30})=1 / 16, P(Z=c+2 / 3 \sqrt{30})=1 / 16, P(Z=c)=7 / 8$. Then $P(X>$ $Z)=P(Z>Y)=P(Y>X)=39 / 64>1 / 2$. As far as we know, it is the first nontransitive dice set, even if the word 'dice' is not mentioned in the paper of Steinhaus and Trybula.

Another construction by Trybula [34, p. 321] is interpreted here as a nontransitive dice set, shown in Table 1. Let $\varphi=\frac{\sqrt{5}+1}{2}$ denote the golden ratio, then its reciprocal can be written as $1 / \varphi=\frac{\sqrt{5}-1}{2} \approx 0.618$.

\begin{tabular}{|l||c|c|c|}
\hline & die 1 & die 2 & die 3 \\
\hline \hline face 1 with probability $1 / \varphi$ & 3 & 2 & 1 \\
\hline face 2 with probability $1-1 / \varphi$ & 0 & 2 & 4 \\
\hline
\end{tabular}

TABLE 1 . Nontransitive dice set by Trybula.

Die 1 beats die 2, die 2 beats die 3 and die 3 beats die 1, each with probability $1 / \varphi>1 / 2$. It is also stated in [30] and shown in [34] that if the random variables $X, Y, Z$ are independent and $P(X>Y)=P(Y>Z)=P(Z>X)$, then $1 / \varphi$ is a sharp upper bound for this probability. If the three probabilities are not necessary equal, then upper bounds can be given for the sum and product of them. Results are extended to $n$ variables in [35].

In the rest of the paper, all faces have the same probabilities and, therefore, probabilities are not denoted explicitly in the tables. 
Moon and Moser [18, p. 531] constructed an example for three teams, each including three players of different skill levels, such that any team is beaten by another team. A comparison between two teams is calculated from all the individual matches played by pairs of players. Their construction can also be interpreted as a nontransitive dice set, where a die is associated with a team, and the numbers written on the faces represent the skill levels of players on that team. One can verify from Table 2 that die 1 beats die 2, die 2 beats die 3 and die 3 beats die 1, with probability 5/9.

\begin{tabular}{|l||c|c|c|}
\hline & die 1 & die 2 & die 3 \\
\hline \hline face 1 & 2 & 1 & 3 \\
\hline face 2 & 6 & 5 & 4 \\
\hline face 3 & 7 & 9 & 8 \\
\hline
\end{tabular}

TABle 2. Nontransitive dice set by Moon and Moser.

Efron's dice set was published by Gardner [12] and, together with dice sets by Schwenk [29] and Rowett [25], is well explained by Peterson[23] and Pegg [22].

Tenney and Foster [33], Székely [31], Batakci and Singer [2] and Epstein [9, pp. 195-199] analyzed a number of dice sets and winning probabilities. Grime [15] constructed and discussed several nontransitive dice sets.

As we saw, the role of the golden ratio had already been realized at the birth of nontransitive dice sets. The reader will probably not be surprised by the fact that Fibonacci numbers are also involved in some of the constructions for nontransitive dice sets. See Savage's paper [27] and recent results by Schaefer and Schweig [28] for more details.

As we have seen in Section 1, van Deventer's dice set realizes $P_{7}$ having property $S_{2}$. In other words, it is a three-player nontransitive dice set: after each of the two opponents pick a die of their choice, the third player will find a die among the remaining dice which beats both opponents' dice. The dice set is originally presented as dice cubes of 6 faces, but they are in fact doubles of 3 faces as in Figure 1. The numbers written on the faces can be divided into three groups 1-7, 8-14 and 15-21, and they are rewritten with additive terms in Table 3. We keep the way of additive writing in the rest of the paper.

\begin{tabular}{|l||c|c|c|c|c|c|c||c|}
\hline & die 0 & die 1 & die 2 & die 3 & die 4 & die 5 & die 6 & add \\
\hline \hline face 1 & 0 & 1 & 2 & 3 & 4 & 5 & 6 & $+1+0 \cdot 7$ \\
\hline face 2 & 4 & 6 & 1 & 3 & 5 & 0 & 2 & $+1+1 \cdot 7$ \\
\hline face 3 & 5 & 2 & 6 & 3 & 0 & 4 & 1 & $+1+2 \cdot 7$ \\
\hline
\end{tabular}

TABLE 3. van Deventer's dice set in additive form.

Note that the dice set presented in [1, p. 377], is equivalent to van Deventer's dice set. Jackson [16] proposed a dice set of five dice for the three-player game. 


\section{Construction of dice Set $\mathbf{D}_{p}$ That Realizes $P_{p}$}

Hereafter, a construction of dice set $\mathbf{D}_{p}$ of $p$ dice is sketched, then it is shown that it realizes $P_{p}$. Remember that $p=4 m+3 \geq 7$ is a prime number. Each die has $p(p-1) / 2$ faces having equal probabilities. The dice set is written as an array of $p(p-1) / 2$ rows and $p$ columns. Each column is associated with a die and faces are organized in rows. Quadratic residues modulo $p$ are denoted by $q_{0}=0, q_{1}=1, q_{2}, q_{3}, \ldots, q_{(p-1) / 2}$ as before. Since $q_{1}=1$ for any $p$, the first $p \times p$ array can be written as in Table 4 .

\begin{tabular}{|l||c|c|c|c|c||c|}
\hline & die 0 & die 1 & die 2 & $\ldots$ & die $p-1$ & add \\
\hline \hline face 1 & $\mathbf{0}$ & $\mathbf{1}$ & $\mathbf{2}$ & $\ldots$ & $\boldsymbol{p}-\mathbf{1}$ & $+0 \cdot p$ \\
\hline face 2 & 1 & 2 & 3 & $\ldots$ & 0 & $+1 \cdot p$ \\
\hline face 3 & 2 & 3 & 4 & $\ldots$ & 1 & $+2 \cdot p$ \\
\hline$\vdots$ & $\vdots$ & $\vdots$ & $\vdots$ & $\ddots$ & $\vdots$ & $\vdots$ \\
\hline face $p$ & $p-1$ & 0 & 1 & $\ldots$ & $p-2$ & $+(p-1) \cdot p$ \\
\hline
\end{tabular}

TABLE 4. The first $p \times p$ array of dice set $\mathbf{D}_{p}$ generated by $q_{1}=1$.

Table 4 is continued by $(p-3) / 2$ arrays, each of which are of size $p \times p$. For a quadratic residue $q_{m} \quad(1 \leq m \leq(p-1) / 2)$, the construction of the key row is as follows. 0 is written on face $(m-1) p+1$ of die $d_{0}$ such that $0 \leq d_{0} \leq p-1$ and $d_{0} \equiv 0 q_{m}(\bmod p)$, that is $d_{0}=0$. Then, for $i=1,2, \ldots, p-1, i$ is written on face $(m-1) p+1$ of die $d_{i}$ such that $0 \leq d_{i} \leq p-1$ and $d_{i} \equiv i q_{m}(\bmod p)$;

Each key face generates $p-1$ additional faces by its shifted copies. The corresponding $p \times p$ array is as in Table 5:

\begin{tabular}{|l||c|c|c|c|c|c|c||c|}
\hline face & die 0 & $\ldots$ & $\operatorname{die} q_{m}$ & $\ldots$ & die $2 q_{m}$ & $\ldots$ & die $p-1$ & add \\
\hline \hline$(m-1) p+1$ & $\boldsymbol{d}_{0}=\mathbf{0}$ & $\ldots$ & $\mathbf{1}$ & $\ldots$ & $\mathbf{2}$ & $\ldots$ & $\ldots$ & $+(m-1) p^{2}$ \\
\hline$(m-1) p+2$ & 1 & $\ldots$ & 2 & $\ldots$ & 3 & $\ldots$ & $\ldots$ & $+(m-1) p^{2}+p$ \\
\hline$(m-1) p+3$ & 2 & $\ldots$ & 3 & $\ldots$ & 4 & $\ldots$ & $\ldots$ & $+(m-1) p^{2}+2 p$ \\
\hline$\vdots$ & $\vdots$ & $\vdots$ & $\vdots$ & $\vdots$ & $\vdots$ & $\ddots$ & $\vdots$ & $\vdots$ \\
\hline$m p-1$ & $p-2$ & $\ldots$ & $p-1$ & $\ldots$ & 0 & $\ldots$ & $\ldots$ & $+m p^{2}-2 p$ \\
\hline$m p$ & $p-1$ & $\ldots$ & 0 & $\ldots$ & 1 & $\ldots$ & $\ldots$ & $+m p^{2}-p$ \\
\hline
\end{tabular}

TABLE 5. The $m$-th $p \times p$ array of dice set $\mathbf{D}_{p}$ generated by $q_{m}$.

The illustrative example $\mathbf{D}_{7}$ is given in the Appendix.

Proposition 2. Dice set $\mathbf{D}_{p}$ constructed in Tables 4-5 realizes $P_{p}$. 
Proof. Note that $P_{p}$ is isomorphic to its reversed version. Consequently, if a dice set realizes the Paley tournament, then it also realizes the reversed Paley tournament after an appropriate reindexing of the dice. An alternative way of reversing a tournament realized by a set of dice, presumably containing only nonnegative values on their faces, is to multiply every value by -1 , then all edges of the tournament reverse. On the other hand, it is sufficient to calculate the odds of die 0 vs. all other dice, due to the rotational symmetry of $P_{p}$. The rest of the proof is built up from four steps:

Lemma 1. The probability that die 0 beats die $i$ is smaller than, equal to, larger than $1 / 2$, if and only if the probability that die 0 beats die $i$, restricted to the cases where their same-indexed faces compete to each other, is smaller than, equal to, larger than $1 / 2$, respectively.

Proof of Lemma 1. Due to the additive terms in the construction $s>t$ implies that face $s$ beats face $t$ for an arbitrary pair of dice. By symmetry, the number of cases when different faces of die 0 and die $i$ compete, is equal to the number of cases when different faces of die $i$ and die 0 compete. The remaining cases are exactly the ones when the same-indexed faces compete.

Lemma 2. The odds of die $0 v s$. die $i$, restricted to the cases when their sameindexed faces compete against each other, are equal to $\left[\sum_{\ell=1}^{(p-1) / 2} v_{\ell, i}\right]$ : $:\left[\sum_{\ell=1}^{(p-1) / 2}\left(p-v_{\ell, i}\right)\right]$, where $v_{\ell, i}$ denotes the value written on $\ell$-th key face of die $i$, and the sums are taken over the $(p-1) / 2$ key faces.

Proof of Lemma 2. First consider only one square array, corresponding to a fixed key face. Let us restrict the dice to this array only. Then the value written on the key face of die $i$ shows the number of cases out of $p$ when die $i$ beats die 0 , because the values follow each other in the same order, and differ only in the rotation. Count the number of cases when die 0 beats die $i$ for every square array corresponding to a key face and take their sum.

Lemma 3. Nonzero quadratic residues are written on key faces of die $i$, where $i$ is a nonzero quadratic residue. Equivalently, quadratic nonresidues are written on key faces of dice $j$, where $j$ is from the set of quadratic nonresidues.

Proof of Lemma 3. Nonzero quadratic residues are written on key faces of dice indexed by nonzero quadratic residues. By analogy, quadratic nonresidues are written on key faces of dice indexed by quadratic nonresidues.

Lemma 4. The probability that die 0 beats die $i$ is greater than $1 / 2$ if and only if $i$ is a quadratic nonresidue. The probability that die 0 beats die $i$ is smaller than $1 / 2$ if and only if $i$ is a nonzero quadratic residue. 
Proof of Lemma 4. Let $i$ be a quadratic nonresidue. It follows from Lemmas 2 and 3 that the odds of die $0 \mathrm{vs}$. die $i$, restricted to the cases when their same-indexed faces compete to each other, are greater than 1 . The number of cases when different faces die 0 vs. die $i$ compete, is equal to the number of cases when different faces of die $i$ vs. die 0 compete. Taking the sum, we get that the odds of die 0 vs. die $i$ are greater than 1 , consequently, the probability that die 0 beats die $i$ is greater than $1 / 2$. The case of nonzero quadratic residue is analogous, the probability that die 0 beats die $i$ is smaller than $1 / 2$. As we see, the crux is in the relation between the sum of quadratic residues and the sum of quadratic nonresidues. It should be also noted that the odds of these dice are never equal. Thus, Lemma 1 could be restated without the case of equality.

Lemma 4 can be specified for any fixed value of $p$, but an explicit formula for the odds is unknown. In case of a minimal tournament $P_{p}$ fulfilling property $S_{k}$ given in Proposition 1, the differences of the sum of quadratic residues and the sum of quadratic nonresidues are $-1 \cdot 7,-1 \cdot 19,-1 \cdot 67,-3 \cdot 331,-7 \cdot 1163$.

Lemmas 1, 2, 3 and 4 complete the proof of Proposition 2.

Remark 1. It follows from the construction that the sum of faces is the same, $\left(p^{5}-2 p^{4}+p^{3}-2 p^{2}+2 p\right) / 8$ for all dice, i.e., each die has the same expected value $\frac{p^{4}-2 p^{3}+p^{2}-2 p+2}{4(p-1)}$.

Proposition 3. If $p>k^{2} 2^{2 k-2}$, then $\mathbf{D}_{p}$ is a nontransitive dice set for $k+1$ players.

Proof. The claim follows from Theorem 2 and Proposition 2.

\section{CONCLUSiOnS}

Research can be continued in several directions, one of them has already been started: finding dice sets that realize $P_{p}$ and have essentially fewer than $p(p-1) / 2$ faces, preliminary results are summarized in [3]. The assumption of the equal probability of the faces can be relaxed, which is supposed to decrease the number of faces. One would expect that replacing discrete random variables with continuous ones requires a different methodology. The historical overview of nontransitive dice refer to the common roots with voting situations, therefore, both areas would benefit from further comparative studies.

\section{ACKNOWLEDGEMENT}

The author read about dice tournaments first on the web pages written by Tim Rowett (Grand Illusions) and Ed Pegg Jr. (Wolfram Research). The author is also grateful to Oskar van Deventer, Jonathan Steinhart, as well as to Lajos Rónyai (Institute for Computer Science and Control, Hungarian Academy of Sciences), Zsolt Tuza (Alfréd Rényi Institute of Mathematics, Hungarian Academy of Sciences), József 
Mala (Corvinus University of Budapest), Tibor Jordán (Eötvös Loránd University), Norihide Tokushige (University of the Ryukyus) and Ryuhei Uehara (Japan Advanced Institute of Science and Technology).

\section{APPEndix: Dice SeT $\mathbf{D}_{7}$}

The construction provided in Section 4 is illustrated for $p=7$.

\begin{tabular}{|l||c|c|c|c|c|c|c||c|}
\hline & die 0 & die 1 & die 2 & die 3 & die 4 & die 5 & die 6 & add \\
\hline \hline face 1 & $\mathbf{0}$ & $\mathbf{1}$ & $\mathbf{2}$ & $\mathbf{3}$ & $\mathbf{4}$ & $\mathbf{5}$ & $\mathbf{6}$ & $+0 \cdot 7$ \\
\hline face 2 & 1 & 2 & 3 & 4 & 5 & 6 & 0 & $+1 \cdot 7$ \\
\hline face 3 & 2 & 3 & 4 & 5 & 6 & 0 & 1 & $+2 \cdot 7$ \\
\hline face 4 & 3 & 4 & 5 & 6 & 0 & 1 & 2 & $+3 \cdot 7$ \\
\hline face 5 & 4 & 5 & 6 & 0 & 1 & 2 & 3 & $+4 \cdot 7$ \\
\hline face 6 & 5 & 6 & 0 & 1 & 2 & 3 & 4 & $+5 \cdot 7$ \\
\hline face 7 & 6 & 0 & 1 & 2 & 3 & 4 & 5 & $+6 \cdot 7$ \\
\hline \hline face 8 & $\mathbf{0}$ & $\mathbf{4}$ & $\mathbf{1}$ & $\mathbf{5}$ & $\mathbf{2}$ & $\mathbf{6}$ & $\mathbf{3}$ & $+7 \cdot 7$ \\
\hline face 9 & 1 & 5 & 2 & 6 & 3 & 0 & 4 & $+8 \cdot 7$ \\
\hline face 10 & 2 & 6 & 3 & 0 & 4 & 1 & 5 & $+9 \cdot 7$ \\
\hline face 11 & 3 & 0 & 4 & 1 & 5 & 2 & 6 & $+10 \cdot 7$ \\
\hline face 12 & 4 & 1 & 5 & 2 & 6 & 3 & 0 & $+11 \cdot 7$ \\
\hline face 13 & 5 & 2 & 6 & 3 & 0 & 4 & 1 & $+12 \cdot 7$ \\
\hline face 14 & 6 & 3 & 0 & 4 & 1 & 5 & 2 & $+13 \cdot 7$ \\
\hline \hline face 15 & $\mathbf{0}$ & $\mathbf{2}$ & $\mathbf{4}$ & $\mathbf{6}$ & $\mathbf{1}$ & $\mathbf{3}$ & $\mathbf{5}$ & $+14 \cdot 7$ \\
\hline face 16 & 1 & 3 & 5 & 0 & 2 & 4 & 6 & $+15 \cdot 7$ \\
\hline face 17 & 2 & 4 & 6 & 1 & 3 & 5 & 0 & $+16 \cdot 7$ \\
\hline face 18 & 3 & 5 & 0 & 2 & 4 & 6 & 1 & $+17 \cdot 7$ \\
\hline face 19 & 4 & 6 & 1 & 3 & 5 & 0 & 2 & $+18 \cdot 7$ \\
\hline face 20 & 5 & 0 & 2 & 4 & 6 & 1 & 3 & $+19 \cdot 7$ \\
\hline face 21 & 6 & 1 & 3 & 5 & 0 & 2 & 4 & $+20 \cdot 7$ \\
\hline
\end{tabular}

TABLE 6. Dice set $\mathbf{D}_{7}$.

Remark 2. Dice set $\mathbf{D}_{7}$ is obviously much less efficient in minimizing the number of faces than van Deventer's dice set, since his dice have 3 faces only (faces 1, 19, 13 in Table 6 are essentially the same as faces 1,2, 3 in Table 3 -they differ only in the additive term).

Remark 3. Table 6 is built up from three $7 \times 7$ arrays, each of which are latin squares if the additional $k \times 7$ is not considered. 


\section{REFERENCES}

[1] N. Alon, G. Brightwell, H. A. Kierstead, A. V. Kostochka, and P. Winkler, "Dominating sets in $k$-majority tournaments," J. Comb. Theory Ser. B, vol. 96, no. 3, pp. 374-387, 2006.

[2] L. Batakci and K. Singer, "Paradox of nontransitive dice," J. of Recreational Mathematics, vol. 34, no. 2, pp. 83-88, 2008.

[3] D. Bednay and S. Bozóki, "Constructions for nontransitive dice sets," in Proceedings of the 8th Japanese-Hungarian Symposium on Discrete Mathematics and its Applications, Veszprém, Hungary, June 4-7, 2013, pp. 15-23.

[4] M. Borowiecki, J. Grytczuk, M. Haluszczak, and Z. Tuza, "Schütte's tournament problem and intersecting families of sets," Comb. Probab. Comput., vol. 12, no. 4, pp. 359-364, 2003.

[5] M. O. v. Deventer, "Phoney dices," Konwakai News, ARM Japan (Academy of Recreational Mathematics), June 1992, also available at www.sztaki.mta.hu/ bozoki/vanDeventer-PhoneyDices-1992.pdf.

[6] G. L. Dirichlet, "Recherches sur diverses applications de l'analyse infinitesimale à la théorie des nombres (première partie)," J. Reine Angew. Math., no. 19, pp. 324-369, 1839.

[7] G. L. Dirichlet, "Recherches sur diverses applications de l'analyse infinitesimale à la théorie des nombres (seconde partie)," J. Reine Angew. Math., no. 21, pp. 1-12, 1840.

[8] G. L. Dirichlet, Vorlesungen über Zahlentheorie, 2nd ed. Braunschweig: Druck und Verlag von Friedrich Vieweg und Sohn, 1871.

[9] R. A. Epstein, The theory of gambling and statistical logic, 2nd ed. Academic Press, 2009.

[10] P. Erdős, "On a problem in graph theory," Math. Gaz., vol. 47, pp. 220-223, 1963.

[11] P. Erdős and A. Rényi, "Asymmetric graphs," Acta Math. Acad. Sci. Hungar., vol. 14, pp. 295-315, 1963.

[12] M. Gardner, "Mathematical games: the paradox of the nontransitive dice and the elusive principle of indifference," Sci. Amer, vol. 223, no. 6, pp. 110-114, 1970.

[13] J. M. Goethals and J. J. Seidel, “Orthogonal matrices with zero diagonal,” Canad. J. Math., vol. 19, no. 5, pp. 1001-1010, 1967.

[14] R. L. Graham and J. H. Spencer, "A constructive solution to a tournament problem," Canad. Math. Bull., vol. 14, no. 1, pp. 45-48, 1971.

[15] J. Grime, "Non-transitive dice," www.singingbanana.com/dice/article.htm.

[16] M. Jackson, "Paradoxes with dice and elections," in Towards Excellence in Mathematics, Proceedings of the 41th annual conference of the Mathematical Association of Victoria, B. Tadich, S. Tobias, C. Brew, B. Beatty, and P. Sullivan, Eds., Monash University, Clayton, Australia, December 2-3, 2004, pp. 208-218.

[17] C. G. J. Jacobi, "Observatio arithmetica de numero classium divisorum quadraticorum formae $y y+a z z$, designante $a$ numerum primum formae $4 n+3$, , J. Reine Angew. Math., no. 9, pp. $189-192,1832$.

[18] J. W. Moon and M. L., "Generating oriented graphs by means of team comparisons," Pac. J. Math., vol. 21 , no. 3, pp. 531-535, 1967.

[19] L. Moser, "A theorem on quadratic residues," Proc. Amer. Math. Soc., vol. 2, no. 3, pp. 503-504, 1951.

[20] I. M. Niven and H. S. Zuckerman, An introduction to the theory of numbers, 3rd ed. Wiley, 1972.

[21] R. E. A. C. Paley, "On orthogonal matrices," J. Math. Phys., vol. 12, pp. 311-320, 1933.

[22] E. Jr. Pegg, “Tournament dice," The Mathematical Association of America, Math Games, 2005, www.mathpuzzle.com/MAA/39-Tournamento20Dice/mathgames_07_11_05.html.

[23] I. Peterson, "Tricky dice revisited," Science News Online, vol. 161, no. 15, 2002, www.sciencenews.org/article/tricky-dice-revisited or archive.is/XWc2o. 
[24] K. B. Reid, A. A. McRae, S. M. Hedetniemi, and S. T. Hedetniemi, "Domination and irredundance in tournaments," Aust. J. Comb., vol. 29, pp. 157-172, 2004.

[25] T. Rowett, "Non-transitive dice set," in Gathering for Gardner V (G4G5), a conference held in honor of Martin Gardner, Atlanta, GA, April 5-7, 2002.

[26] H. Sachs, "Über selbstkomplementäre Graphen,” Publ. Math. Debrecen, vol. 9, pp. 270-288, 1962.

[27] R. P. Savage, “The paradox of nontransitive dice," Am. Math. Mon., vol. 101, no. 5, pp. 429-436, 1994.

[28] A. Schaefer and J. Schweig, "Balanced non-transitive dice," manuscript, www. math.okstate. edu/ jayjs/balanced082812.pdf.

[29] A. J. Schwenk, "Beware of geeks bearing gifts," Math Horizons, vol. 7, pp. 10-13, 2000.

[30] H. Steinhaus and S. Trybula, "On a paradox in applied probabilities," Bull. Acad. Polon. Sci. Math. Astronom. Phys., vol. 7, pp. 67-69, 1959.

[31] G. J. Székely, Paradoxes in probability theory and mathematical statistics. D. Reidel Publishing Company, Dordrecht, Holland and Akadémiai Kiadó, Budapest, Hungary, 1987.

[32] E. Szekeres and G. Szekeres, "On a problem of Schütte and Erdős," Math. Gaz., vol. 49, no. 369, pp. 290-293, 1965.

[33] R. L. Tenney and C. C. Foster, "Non-transitive dominance," Math. Mag., vol. 49, no. 3, pp. 115120.

[34] S. Trybula, "On the paradox of three random variables," Zastos. Mat., vol. 5, pp. 321-332, 1961.

[35] S. Trybula, "On the paradox of $n$ random variables," Zastos. Mat., vol. 8, pp. 143-156, 1965.

[36] J. Tyszkiewicz, "A simple construction for tournaments with every $k$ players beaten by a single player," Am. Math. Mon., vol. 107, no. 1, pp. 53-54, 2000.

Author's address

S. Bozóki

Institute for Computer Science and Control, Hungarian Academy of Sciences (MTA SZTAKI), Laboratory on Engineering and Management Intelligence, Research Group of Operations Research and Decision Systems, 13-17 Kende St., 1111 Budapest, Hungary

E-mail address: bozoki.sandoresztaki.mta.hu 